\title{
Parametric Population Representation of Retinal Location: Neuronal Interaction Dynamics in Cat Primary Visual Cortex
}

\author{
Dirk Jancke, ${ }^{1}$ Wolfram Erlhagen, ${ }^{1}$ Hubert R. Dinse, ${ }^{1}$ Amir C. Akhavan, ${ }^{1,2}$ Martin Giese, ${ }^{1}$ Axel Steinhage, ${ }^{1}$ and \\ Gregor Schöner ${ }^{3}$
}

1/nstitut für Neuroinformatik, Theoretische Biologie, Ruhr-Universität, D-44780 Bochum, Germany, ${ }^{2}$ Keck Center for

Integrative Neuroscience, University of California, San Francisco, California 94143, and ${ }^{3}$ Centre de Recherche en

Neurosciences Cognitives, Centre National de la Recherche Scientifique, F-13402 Marseille, France

\begin{abstract}
Neuronal interactions are an intricate part of cortical information processing generating internal representations of the environment beyond simple one-to-one mappings of the input parameter space. Here we examined functional ranges of interaction processes within ensembles of neurons in cat primary visual cortex. Seven "elementary" stimuli consisting of small squares of light were presented at contiguous horizontal positions. The population representation of these stimuli was compared to the representation of "composite" stimuli, consisting of two squares of light at varied separations. Based on receptive field measurements and by application of an Optimal Linear Estimator, the representation of retinal location was constructed as a distribution of population activation (DPA) in visual space. The spatiotemporal pattern of the DPA was investigated by obtaining the activity of each neuron for a sequence of time intervals. We found that the DPA of composite stimuli deviates from the
\end{abstract}

superposition of its components because of distancedependent (1) early excitation and (2) late inhibition. (3) The shape of the DPA of composite stimuli revealed a distancedependent repulsion effect. We simulated these findings within the framework of dynamic neural fields. In the model, the feedforward response of neurons is modulated by spatial ranges of excitatory and inhibitory interactions within the population. A single set of model parameters was sufficient to describe the main experimental effects. Combined, our results indicate that the spatiotemporal processing of visual stimuli is characterized by a delicate, mutual interplay between stimulusdependent and interaction-based strategies contributing to the formation of widespread cortical activation patterns.

Key words: cat; interaction; neural ensembles; neural field; optimal linear estimator; population code; population dynamics; receptive field; striate cortex; visual field
During the recent years neurons of the visual cortex have been extensively investigated according to a diversity of feature attributes. In search of optimal stimulus conditions, they were classified with respect to differing receptive field (RF) properties. However, RFs can exhibit complex, nonpredictive behavior dependent on further variations of the stimulus parameters. In addition, these complex spatiotemporal response properties can be modified by stimulation displaced from the RF center or from outside the classical RF (Allman et al., 1985; Dinse, 1986; Gilbert and Wiesel, 1990; Sillito et al., 1995). These observations were explained with results from anatomical and physiological studies revealing extensive long-range horizontal intracortical connections (Fisken et al., 1975; Creutzfeldt et al., 1977; Gilbert and Wiesel, 1979, 1990; Kisvárday and Eysel, 1993; Bringuier et al., 1999). Accordingly, optical imaging techniques demonstrated that the cortical processing of even very small objects is associated with a widespread pattern of cortical population activation (Grinvald et al., 1994; Godde et al., 1995).

Neural population analysis refers to the notion that large ensembles of neurons contribute to the cortical representation of

\footnotetext{
Received March 31, 1999; revised July 21, 1999; accepted Aug. 2, 1999.

This work was supported by grants from the Deutsche Forschungsgemeinschaft (Scho 336/4-2 to G.S. and Di 334/5-1,3 to H.D.). We thank Dr. Alexa Riehle and Annette Bastian for discussion, Dr. Christoph Schreiner for helpful comments on an earlier version of this manuscript, and David Kastrup for proofreading.

Correspondence should be addressed to Dr. Dirk Jancke, Institut für Neuroinformatik, Theoretische Biologie ND 04, Ruhr-Universität Bochum, D-44780 Bochum, Germany.

Copyright (C) 1999 Society for Neuroscience 0270-6474/99/199016-13\$05.00/0
}

sensory or motor parameters. Early formulations of this idea (Erickson, 1974) conceived of the representation of complex stimuli in terms of elementary feature detectors simply as a combination of the simultaneous levels of their activation. In primary motor cortex, ensembles of neurons broadly tuned to the direction of movement have been shown to accurately represent the current value of that parameter (Georgopoulos et al., 1986, 1993). These observations inspired renewed attempts to investigate sensory representations in terms of population codes (Steinmetz et al., 1987; Lee et al., 1988; Vogels, 1990; Young and Yamane, 1992; Wilson and McNaughton, 1993; Nicolelis and Chapin, 1994; Ruiz et al., 1995; Jancke et al., 1996; Kalt et al., 1996; Zhang, 1996; Groh et al., 1997; Sugihara et al., 1998; Zhang et al., 1998) and triggered theoretical work examining the formal basis of coding by populations of neurons (Gielen et al., 1988; Vogels, 1990; Zohary, 1992; Gaal, 1993a,b; Seung and Sompolinsky, 1993; Anderson, 1994a,b; Salinas and Abbott, 1994; Giese et al., 1997; Pouget et al., 1998; Zemel et al., 1998; Zhang et al., 1998).

In this paper we studied how small visual stimuli can be represented by the joint activation of a population of neurons in cat primary visual cortex and how neurons within such a population interact in terms of a common metric dimension, in our case, in visual space.

In a first step, we attempted to extract the contribution of neurons to the representation of the location of small squares of light, which we called "elementary" stimuli (Fig. $1 A$ ). We therefore constructed distributions of population activation (DPAs) defined in the visual field that can be regarded as a subspace of 
A

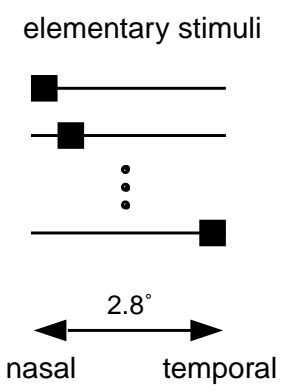

D
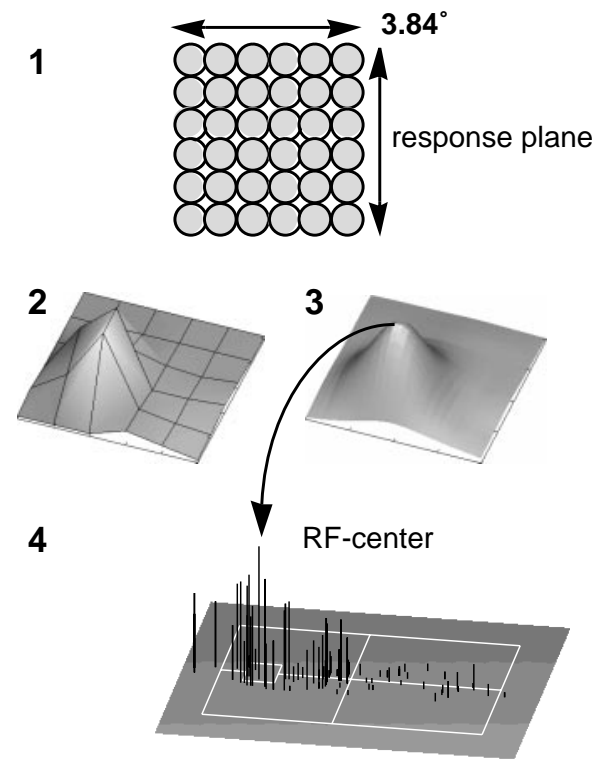

4
C

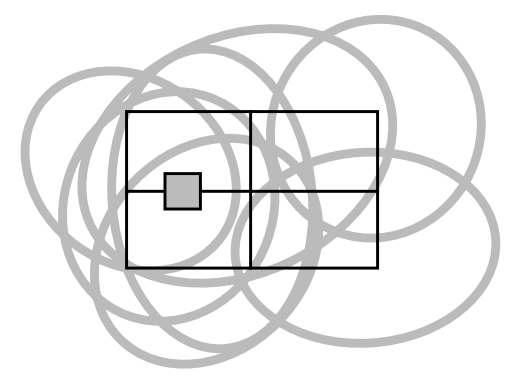

E

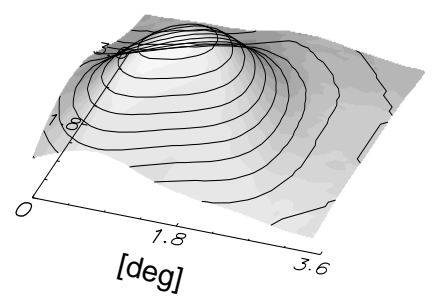

F

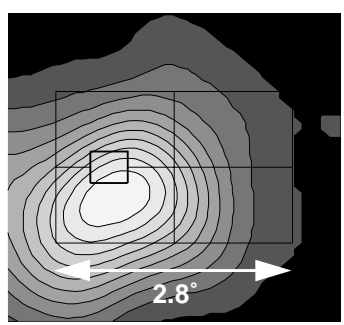

Figure 1. A, Schematic illustration of the elementary stimuli (squares of light, $0.4 \times 0.4^{\circ}$ ) presented at seven horizontally shifted positions within the foveal representation of the visual field. $B$, Composite stimuli were assembled from combinations of the elementary stimuli and were presented at six different separation distances of $0.4-2.4^{\circ}$. The left stimulus component was kept at a fixed nasal position. $C$, Illustration of the noncentered field approach. Stimuli, indicated by the small gray square, were presented independent of the locations of the RFs of the measured neurons (schematically illustrated by gray ellipses). The frame with the cross-hair illustrates the analyzed portion of the visual space $(2.8 \times 2.0)$. $D-F$, Illustration of the Gaussian interpolation method to construct the DPA. D1, The grid of stimuli used (36 circles, each $0.64^{\circ}$ in diameter) to measure the RF profile of each neuron was centered on the hand-plotted RF (response plane technique). D2, The RF profile constructed from responses to this stimulus grid was smoothed (D3) with a Gaussian filter (width, $0.64^{\circ}$ ). The RF center was determined as the location of the centroid of this smoothed RF profile. D4, The contribution of each cell to the population representations was always centered on this location and was weighted with the current firing rate of the neuron, illustrated as vertical bars of varying length. This weighting factor was normalized to the maximal firing rate of each neuron. $E$, The DPA was obtained by Gaussian interpolation (width, $0.6^{\circ}$ ) of the weighted firing rates and by a subsequent convolution with an unweighted Gaussian (width, $0.64^{\circ}$ ). $F$, View of the distribution of population activation using gray levels to indicate activation. The location of the stimulus is indicated by the small square outlined in black together with the stimulus frame. In a second approach, one-dimensional DPAs were derived by means of an OLE; see Materials and Methods and Figure $2 C$.

the potentially high-dimensional space of visual stimulus attributes. The second step consisted of projecting the neural responses to "composite" stimuli assembled from two squares of light at varied separations (Fig. $1 B$ ) onto this subspace by analyzing DPAs weighted with the responses to composite stimuli. Distance-dependent deviations of the DPAs from the superposition of the corresponding elementary components reveal insight into interaction processes within the representation of retinal location at the population level. Such interaction may arise from recurrent connectivity within the cortical area as well as from recurrence within the network providing the sensory input. A neural field model explicates how such mechanisms contribute to the evolution of cortical activation within ensembles of neurons.

\section{MATERIALS AND METHODS}

\section{Experimental setup}

Animals and preparation. Electrophysiological recordings from a total of 178 cells were made extracellularly in the foveal representation of area 17 in 20 adult cats of both sexes. Animals were initially anesthetized with Ketanest (15 mg/kg body weight, i.m.; Parke-Davis, Courbevoie, France) and Rompun (1 mg/kg, i.m.; Bayer, Wuppertal, Germany). Additionally, atropin $(0.1 \mathrm{mg} / \mathrm{kg}$, s.c.; Braun) was given. After intubation with an endotracheal tube, animals were fixated in a stereotactic frame. During surgery and recording, anesthesia was maintained by artificial respiration with a mixture of $75 \% \mathrm{~N}_{2} \mathrm{O}$ and $25 \% \mathrm{O}_{2}$ and by application of sodium pentobarbital (Nembutal, $3 \mathrm{mg} \cdot \mathrm{kg}^{-1} \cdot \mathrm{hr}^{-1}$, i.v.; Ceva). Treatment of all animals was within the regulations of the National Institution of Health Guide and Care for Use of Laboratory Animals (1987). Animals were paralyzed by continuous infusions of gallamine triethiodide $(2 \mathrm{mg} / \mathrm{kg}$, i.v. 
bolus; $2 \mathrm{mg} \cdot \mathrm{kg}^{-1} \cdot \mathrm{hr}^{-1}$ i.v., Sigma, St. Louis, MO). In addition, 5\% glucose in physiological Ringer's solution was continuously infused (3 $\mathrm{ml} / \mathrm{hr}$; Braun). Heart rate, intratracheal pressure, expired $\mathrm{CO}_{2}$, body temperature, and EEG were monitored during the entire experiment. Respiration was adjusted for an end-tidal $\mathrm{CO}_{2}$ between 3.5 and $4.0 \%$. The body temperature was kept at $37.5^{\circ} \mathrm{C}$ by means of a feedbackcontrolled heating pad. Contact lenses with artificial pupils $(3 \mathrm{~mm}$ diameter) were used to cover the eyes, which were frequently rinsed with artificial eye liquid (Liquifilm; Pharm-Allergan). Pupils were dilated by atropine $(5 \mathrm{mg} / \mathrm{ml})$, and nictitating membranes were retracted by norepinephrine (Neosynephrin-POS, $50 \mathrm{mg} / \mathrm{ml}$; Ursapharm). The bone and dura mater were removed over the central representation of area 17 in the left hemisphere. The exposed cortex was covered with heavy silicone oil. At the end of the experiments, animals were killed with an overdose of sodium pentobarbital.

Data acquisition. We recorded responses of single units in the foveal representation in area 17 of the left hemisphere. Stimuli were always presented to the contralateral eye. Recordings were performed simultaneously with two or three glass-coated platinum electrodes (resistance between 3.5 and $4.5 \mathrm{M} \Omega$; Thomas Recording), which were advanced with a microstepper. The bandpass-filtered $(500-3000 \mathrm{~Hz})$ electrode signals were fed into spike sorters based on an on-line principle component analysis (Gawne and Richmond, National Institutes of Health, Bethesda, MD). Their output TTL-pulses were stored on a personal computer (PC) with a time resolution of $1 \mathrm{msec}$. Raw analog recordings were displayed on oscilloscopes and on audio monitors. Digitized neural responses were displayed as poststimulus time histograms (PSTHs) online during the recording sessions.

Data were analyzed off-line in the Interactive Data Language graphical environment (Research Systems, Inc.).

Visual stimulation. Stimuli were displayed on a PC-controlled 21 inch monitor $(120 \mathrm{~Hz}$, noninterlaced) positioned at a distance of $114 \mathrm{~cm}$ from the animal.

An identical set of common stimuli was presented to all neurons: (1) elementary stimuli (Fig. $1 \mathrm{~A}$ ), small squares of light (size, $0.4 \times 0.4^{\circ}$ ), were flashed at one of seven different horizontally contiguous locations within a fixed foveal reference frame; and (2) composite stimuli (Fig. $1 B$ ), two simultaneously flashed squares of light, were separated by distances that varied between 0.4 and $2.4^{\circ}$. Each stimulus was flashed for $25 \mathrm{msec}$. The interstimulus interval (ISI) was $1500 \mathrm{msec}$. There were a total of 32 repetitions of each stimulus, arranged in pseudorandom order across the different conditions. Stimuli had a luminance of $0.9 \mathrm{~cd} / \mathrm{m}^{2}$ against a background luminance of $0.002 \mathrm{~cd} / \mathrm{m}^{2}$. The retinal position of these common stimuli was constant, irrespective of the RF location of individual neurons (non-RF-centered approach illustrated in Fig. $1 C, D 4)$.

The profile of each individual RF was assessed quantitatively with a separate set of stimuli, consisting of small dots of light (diameter, $0.64^{\circ}$ ) that were flashed in pseudorandom order (20 times) for $25 \mathrm{msec}$ (ISI, $1000 \mathrm{msec}$ ) on the 36 locations of an imaginary $6 \times 6$ grid, centered over the hand-plotted RF (response plane technique, Fig. 1D1). To control for eye drift, RF profiles were repeatedly measured during each recording session.

\section{Construction of the DPA}

The general idea behind constructing a population distribution is to extract the contributions of neurons to the representation of a particular stimulus parameter. To obtain entire distributions that are defined for visual field location, two types of analysis were applied: (1) based on the measured RF profiles (Fig. 1D1,D2), the calculated RF centers (Fig. $1 D 3)$ served to construct two-dimensional DPAs by interpolating the normalized firing rates of each contributing neuron with a Gaussian profile (cf. Anderson, 1994a,b, for a related attempt) (Fig. 1E,F); and (2) to minimize the reconstruction error for the elementary stimulus conditions, we extended the Optimal Linear Estimator (OLE) (Salinas and Abbott, 1994), resulting in one-dimensional DPAs (Fig. 2C).

Constructing two-dimensional DPAs by Gaussian interpolation. For each location on the $6 \times 6$ grid, an average response strength was determined for each cell by averaging the firing rate in the time interval between 40 and $65 \mathrm{msec}$ after stimulus onset corresponding to the peak responses in the PSTHs. RF profiles were obtained (Fig. 1D2) and smoothed by convolution with a Gaussian profile in two dimensions (half width, $0.64^{\circ}$; Fig. 1D3). The center of the RF of each cell was then computed as the center of mass of that part of the RF profile that exceeded half of the maximal firing rate.
The firing rate, $f_{\mathrm{n}}(s, t)$ of neuron number $n$ to stimulus number $s$ was defined as the firing rate in a $10 \mathrm{msec}$ time interval beginning at time $t$ after stimulus onset, averaged over 32 stimulus repetitions. Spontaneous activity, $b_{\mathrm{n}}$, was estimated as the mean firing rate accumulated over nonstimulus trials. For the purpose of constructing the population representation, the firing rate of each cell was normalized to its maximum firing rate, $m_{\mathrm{n}}$, over all stimuli used to measure the response planes and during any single $10 \mathrm{msec}$ bin in the time interval from stimulus onset to $100 \mathrm{msec}$ after stimulus onset. This normalized firing rate:

$$
F_{n}(s, t)=\frac{f_{n}(s, t)-b_{n}}{m_{n}-b_{n}}
$$

was always well defined and positive (Fig. 1D4).

The normalized firing rates, $F_{\mathrm{n}}(s, t)$, were depicted at the position of the calculated RF center of each neuron. For interpolation of the data points, the width of the Gaussian profile was chosen equal to $0.6^{\circ}$ in visual space (approximately corresponding to the average RF width of all neurons recorded) (Fig. 2A). To correct for uneven sampling of visual space by the limited number of RF centers, the distribution was normalized by dividing by a density function, which was simply the sum of unweighted Gaussian profiles (width, $0.64^{\circ}$ ) centered on all RF centers. This procedure is illustrated in Figure 1, $E$ and $F$.

Deriving the optimal linear estimator for the DPA. An optimal estimation of the DPA is based on the responses to elementary stimuli. For each stimulus position $s_{\mathrm{i}}$, the DPA, $\hat{U}_{\mathrm{i}}\left(s_{\mathrm{k}}\right)$, is constructed as a linear combination of contributions from each neuron $(n=1, \ldots, N)$ :

$$
\hat{U}_{i}\left(s_{k}\right)=\sum_{n=1}^{N} c_{n}\left(s_{k}\right) f_{n}\left(s_{i}\right)
$$

The number $M$ of sample points $s_{\mathrm{k}}$ determines the degree of resolution with which the DPAs are sampled. The contribution of each neuron is a basis function, $c_{\mathrm{n}}\left(s_{\mathrm{k}}\right)$, to be determined by optimization, multiplied with the firing rate, $f_{\mathrm{n}}\left(s_{\mathrm{i}}\right)$, averaged over the time interval between 40 and 65 msec after stimulus onset. The desired form of the DPA representation of these stimuli is explicitly chosen as a Gaussian, $U_{\mathrm{i}}\left(s_{\mathrm{k}}\right)$, centered on each stimulus position, $s_{\mathrm{i}}$ :

$$
U_{i}\left(s_{k}\right)=\exp \left(-\frac{\left(s_{k}-s_{i}\right)^{2}}{2 \sigma^{2}}\right) \text { with } s_{k} \in\left[s_{1}-\sigma, s_{7}+\sigma\right], k=1 \ldots M .
$$

The width $\sigma=0.6^{\circ}$ was chosen such that $U_{\mathrm{i}}\left(s_{\mathrm{k}}\right)$ fits to the average RF profile of all measured neurons (Fig. $2 A$ ). To determine the basis functions we minimize the average reconstruction error $\Sigma_{i}\left(\hat{U}_{i}\left(s_{\mathrm{k}}\right)-\right.$ $\left.U_{i}\left(s_{k}\right)\right)^{2}$ (Seung and Sompolinsky, 1993; Salinas and Abbott, 1994; Pouget et al., 1998), which leads to:

$$
c_{n}\left(s_{k}\right)=\sum_{m=1}^{N} L_{m}\left(s_{k}\right) Q_{n m}^{-1} .
$$

Here, $Q_{n m}$ is the correlation matrix between the firing rates of neurons $n$ and $m$ for all stimuli:

$$
Q_{n m}=\sum_{i=1}^{7} f_{n}\left(s_{i}\right) f_{m}\left(s_{i}\right)
$$

and $L_{m}\left(s_{k}\right)$ is:

$$
L_{m}=\sum_{i=1}^{7} U_{i}\left(s_{k}\right) f_{m}\left(s_{i}\right)
$$

This amounts to an OLE for a vector-valued stimulus parameter (Salinas and Abbott, 1994).

This estimator can then be extrapolated to obtain time-resolved DPAs by replacing the averaged firing rate $f_{\mathrm{n}}\left(s_{\mathrm{i}}\right)$ in Equation 2 by the firing rate in a particular time interval. The coefficients $c_{n}\left(s_{k}\right)$, by contrast, remain fixed. This extrapolated DPA is the basis for investigating the nonlinear interaction effects within the composite stimulus paradigm. We compare the superpositions: 
A

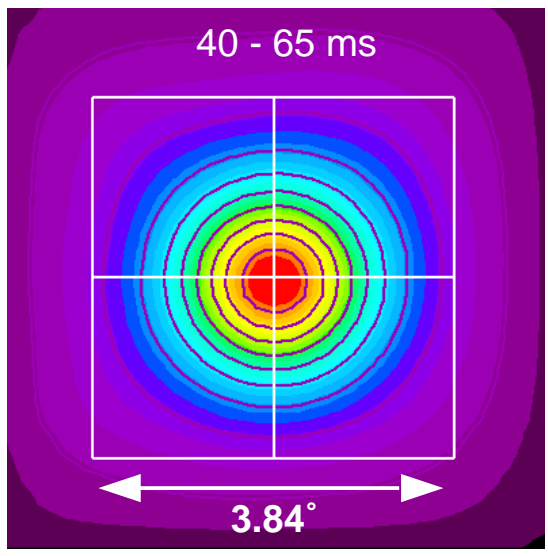

C

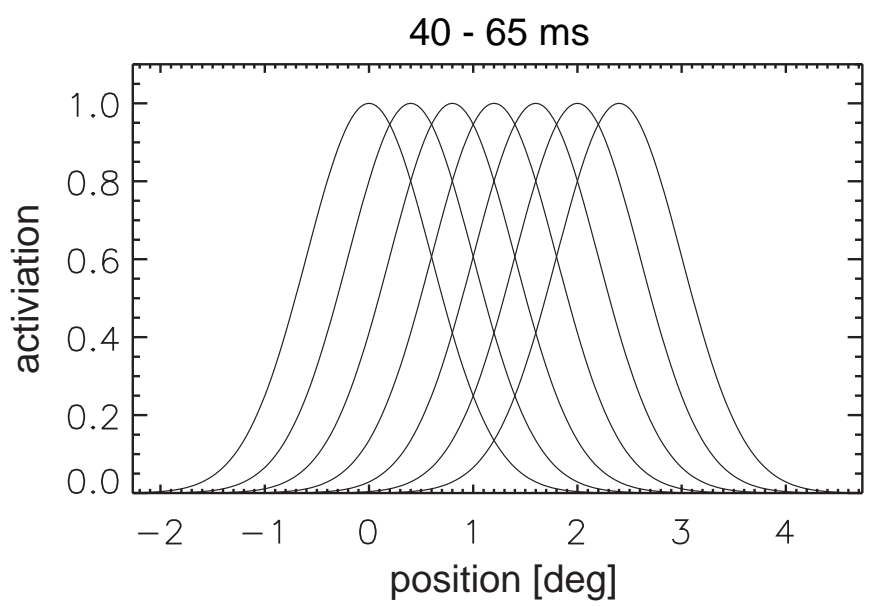

B

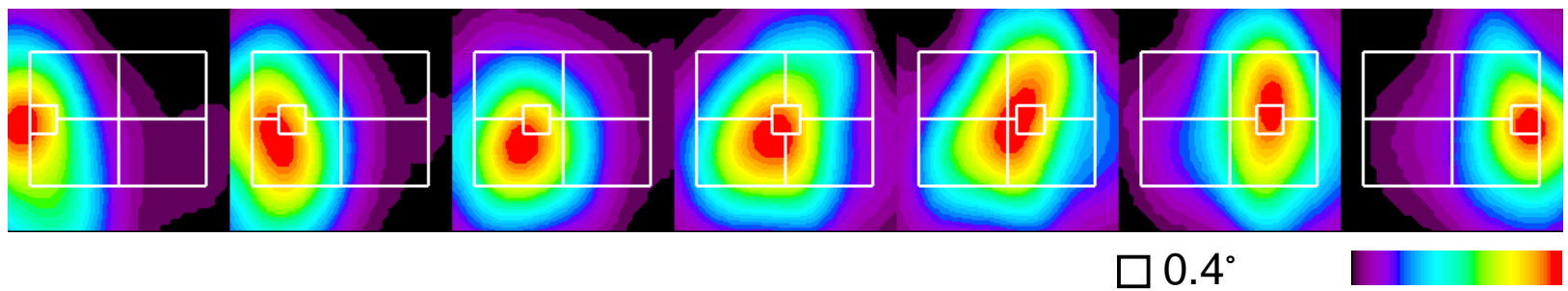

Figure 2. A, Average RF, corresponding to the tuning for location, of all 178 recorded neurons. Based on the peak responses in the PSTHs (40-65 msec after stimulus onset) each RF profile was smoothed by convolution with a Gaussian in two dimensions (width, $0.64^{\circ}$ ). RF centers were derived by calculating the centroid of each profile (compare Fig. 1D3). For summation, the smoothed profiles were added with respect to their RF centers. The SD was $0.6^{\circ}$ (calculated for that part of the resulting average RF profile, which exceeded half of the maximal amplitude). This value of average RF width matches the typical RF sizes found in area 17 of the cat (Orban, 1984). The vertical arrow indicates the spatial extension in terms of visual field coordinates. $B$, Population representations of the elementary stimuli computed as two-dimensional DPAs over visual space after Gaussian interpolation (compare Fig. 1). The construction was based on the activity of 178 neurons. DPAs were computed in the time interval between 40 and 65 msec after stimulus onset corresponding to the peak responses in the PSTHs. The activation level is shown in a color scale normalized to maximal activation separately for each stimulus (calibration bar at bottom right). Red indicates high levels of activation. The frame outlined in white depicts the area of the visual field investigated as described in Figure $1 C$. In addition, the stimulus is shown as a square outlined in white. Note that for each stimulus the focal zone of activation is approximately centered on the stimulus location. $C$, DPAs derived by means of an OLE for all seven elementary stimuli used. DPAs were assumed as Gaussian profiles centered on each respective stimulus position. As in the interpolation procedure, neural activity was integrated between 40 and $65 \mathrm{msec}$ after stimulus onset. The width of the estimated Gaussian was chosen $0.6^{\circ}$ to match the average RF width (tuning curve) of all neurons measured (compare Fig. $2 A$ ). The maxima of the OLE-derived distributions were aligned accurately on the position of each stimulus.

$$
\hat{U}_{i j}^{\text {sup }}\left(s_{k}, t\right)=\hat{U}_{i}\left(s_{k}, t\right)+\hat{U}_{j}\left(s_{k}, t\right)
$$

of the time-resolved DPAs for two elementary stimuli $s_{\mathrm{i}}$ and $s_{\mathrm{j}}$ with the time-resolved DPAs of composite stimuli

$$
\hat{U}_{i j}^{\text {meas }}\left(s_{k}, t\right)=\sum_{n=1}^{N} c_{n}\left(s_{k}\right) f_{n}\left(s_{i}, s_{j}, t\right) .
$$

$\hat{U}_{i j}^{\text {meas }}\left(s_{k}, t\right)$ is the extrapolated DPA that is based on replacing the rate $f_{n}\left(s_{i}\right)$ in Equation 2 by the firing rates $f_{n}\left(s_{i}, s_{j}, t\right)$ that are observed in response to the corresponding composite stimulus.

\section{RESULTS}

\section{Experimental results}

\section{Distributions of population activation of elementary stimuli}

We constructed DPAs in response to a set of small squares of light that only differ in their position along a virtual horizontal line and that we termed elementary stimuli. The DPAs were defined in visual space and were based on single cell responses from 178 neurons recorded in the foveal representation of cat area 17. To obtain DPAs, we made use of two different approaches: (1) in a two-dimensional Gaussian interpolation procedure, the RF cen- ters were weighted with the normalized firing rate of each neuron (Fig. $1 D-F)$. Corresponding to the average RF profile of all neurons recorded (compare Fig. $2 A$ ), the width of the Gaussian was chosen uniformly to $0.6^{\circ}$; and (2) in addition, based on the assumption that the representation of visual location can be considered as a function of activation in parameter space, we minimized the error for reconstructing one-dimensional distributions using the OLE procedure. This method is optimal in the sense that it extracts the available information from the firing rates under the condition of a least square fit.

As a reference, we calculated DPAs in the time interval between 40 and $65 \mathrm{msec}$ after stimulus onset corresponding to the peak responses in the PSTHs. Both approaches yielded equivalent results. The DPAs were monomodal and centered onto each respective visual field position. For each stimulus, Figure $2 B$ depicts the two-dimensional DPAs of all seven elementary stimuli constructed by Gaussian interpolation. Figure $2 C$ shows the OLEderived one-dimensional DPAs. The spatial arrangement of activity within these distributions implies that neurons in primary visual cortex contribute as an ensemble to the representation of 


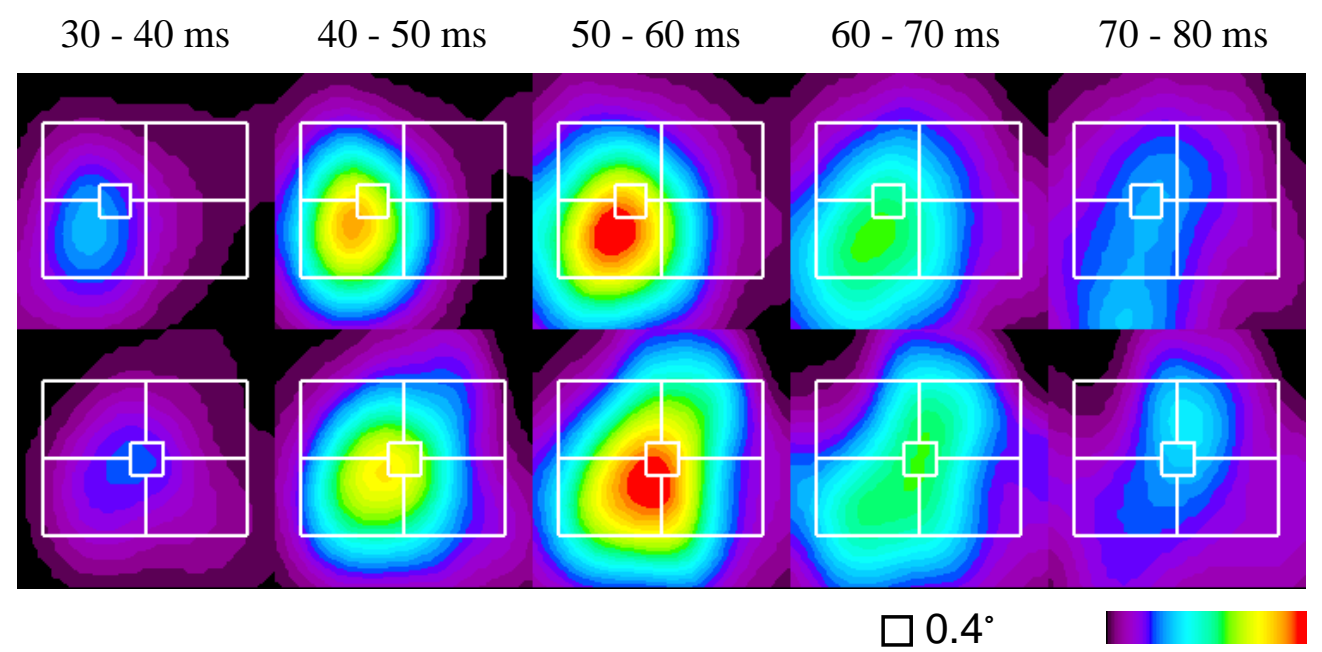

Figure 3. Two-dimensional DPAs of adjacent elementary stimuli (top and bottom) derived by Gaussian interpolation. The DPAs were obtained for consecutive intervals of $10 \mathrm{msec}$ duration covering the period from 30 to $80 \mathrm{msec}$ after stimulus onset. Same conventions as in Figure $2 B$. Each example was normalized separately. As for the OLE-derived DPAs (compare Fig. 4), the distributions grow and decay gradually, and their maximum is always located near the position of the stimulus. Although the two stimuli are at neighboring locations, differences of the spatial representations are apparent throughout the time course of the response. For all elementary stimuli, the average latency of maximal activation was $54 \pm 4$ msec.

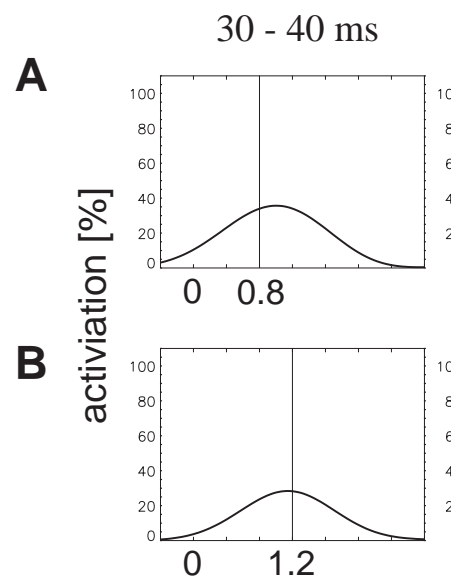

$40-50 \mathrm{~ms}$
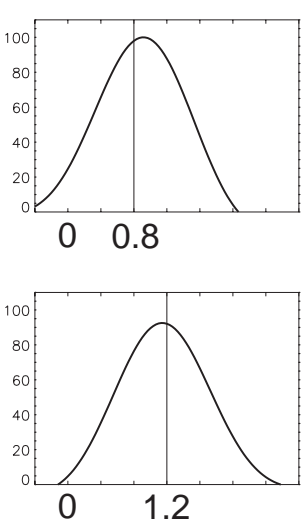

$50-60 \mathrm{~ms}$
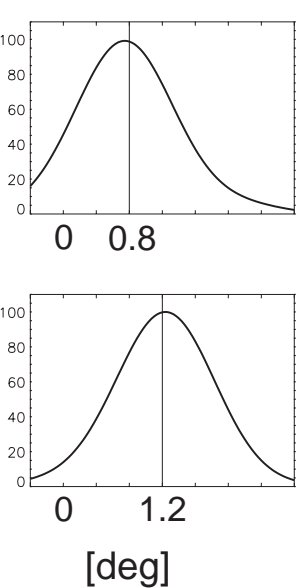

$60-70 \mathrm{~ms}$
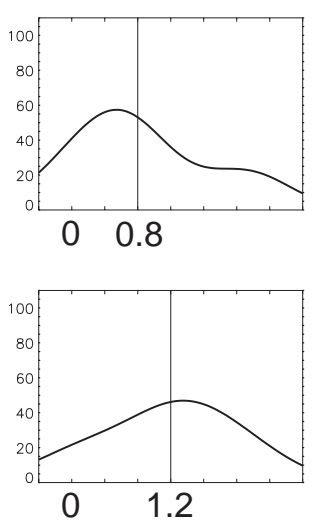

$70-80 \mathrm{~ms}$
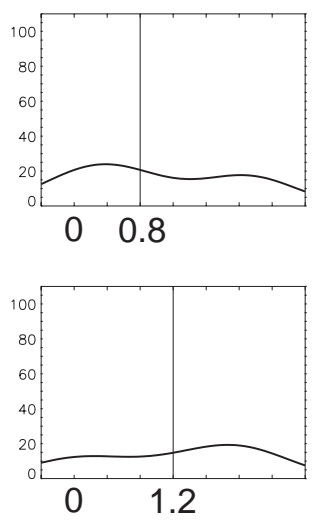

Figure 4. The temporal evolution of two OLE-derived DPAs of the same elementary stimuli (A, B, vertical lines indicate position) as shown in Figure 3. The DPAs are depicted in $10 \mathrm{msec}$ time intervals covering the period from 30 to $80 \mathrm{msec}$. The distributions grow and decay, gradually reaching maximum activity at $53 \pm 5 \mathrm{msec}$ (average of all seven elementary stimuli) after stimulus onset. The position of the maximum of each distribution closely approximates the stimulus position of the elementary stimulus throughout the time course of the neural population response, yet less accurately in the late time epoch.

visual field location, although the RF of each neuron might be broadly tuned to stimulus location.

For extrapolation, DPAs were obtained by replacing the neural activity observed in other time intervals or in response to composite stimuli.

\section{Temporal evolution of the DPAs of elementary stimuli}

The main emphasis of this study was to explore cortical interaction processes. It appears conceivable that such processes can be traced during the entire temporal structure of neuron responses because of differences of time constants of excitatory and inhibitory contributions (Bringuier et al., 1999) and because of timedelayed feedback (Dinse et al., 1990). Accordingly, as an important prerequisite, time-resolved DPAs were constructed for a number of subsequent time intervals after stimulus onset using the firing rates within each time slice as weights. Figures 3 and 4 illustrate the temporal evolution of the DPAs from 30 to $80 \mathrm{msec}$ after stimulus onset for two selected elementary stimuli. There is a remarkable spatial coherence of activity within the ensemble. The gradual build-up and decay of activation were quite uniform across the distributions of all elementary stimuli.

On average, the DPAs constructed by Gaussian interpolation reached maximal level of activation $54 \pm 4$ msec after stimulus onset as compared to $53 \pm 5 \mathrm{msec}$ for the OLE-derived DPAs (see Fig. 9B). To quantitatively assess the accuracy with which the DPAs represent the location of the elementary stimuli position during the entire time course of responses analyzed (30-80 msec), we compared the position of the maximum of each DPA to the respective stimulus position. Figure 5 plots these constructed positions against the real stimulus positions. Results from both reconstruction methods revealed that the DPAs represent stimu- 
A

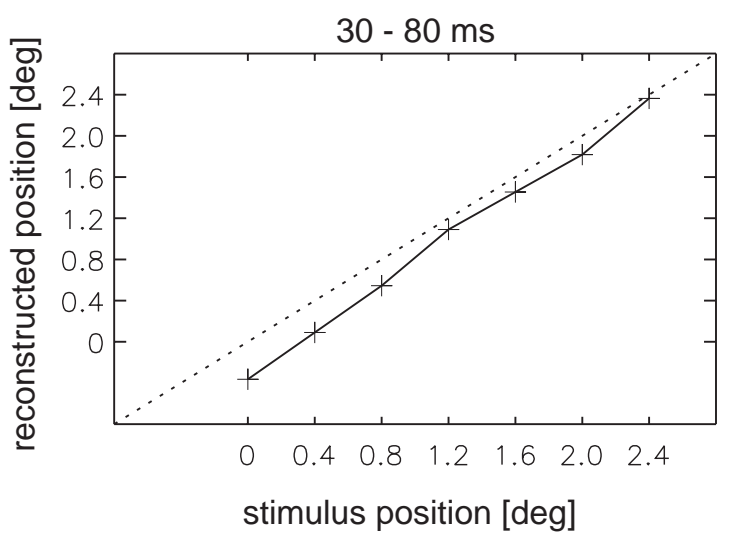

B

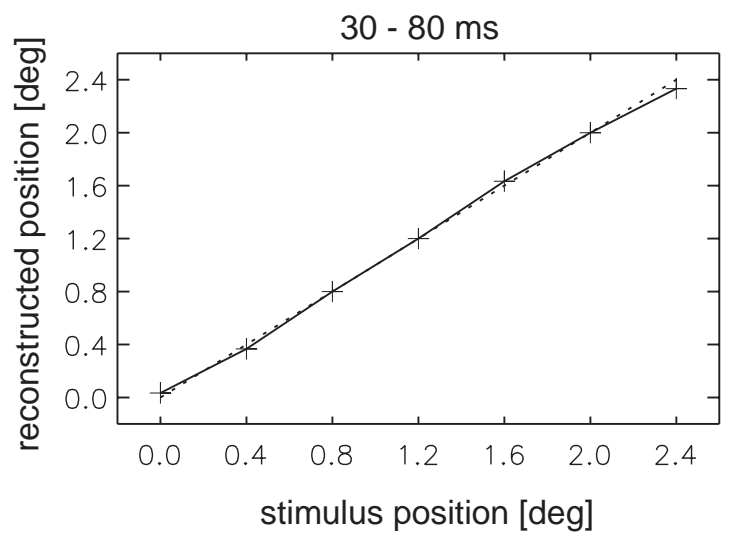

Figure 5. Constructed versus real position of the elementary stimuli using the averaged spike activity during the entire time course of responses $(30-80 \mathrm{msec})$. The position of the maximum of the DPA is shown for the seven elementary stimuli as a function of the real stimulus position. The dotted line indicates the perfect match between estimated and real stimulus position. $A$, The two-dimensional distributions as shown in Figure $2 B$ were summed along the vertical axis to obtain the horizontal position of the maximum only. Using the Gaussian interpolation method, stimulus position can be estimated as well, but less accurate as compared to the OLE-derived DPAs (average deviation for all elementary stimuli, $\left.0.20 \pm 0.11^{\circ}\right) \cdot B$, Examination of the OLE-derived DPAs proved that the estimator accounts for a high spatial accuracy during the entire neural activation (average deviation for all elementary stimuli, $0.02 \pm 0.02^{\circ}$ ).

lus position during this investigated time window. The average deviation was $0.20 \pm 0.11^{\circ}$ for the interpolated DPAs and $0.02 \pm$ $0.02^{\circ}$ for the distributions based on optimal estimation. The optimal estimation allowed us to avoid reconstruction errors but might suppress systematic errors that were revealed by the interpolation procedure (Fig. $5 A$ ). Interestingly, in a recent psychophysical study, briefly presented stimuli have been found to be mislocalized more foveally (Müsseler et al., 1999).

\section{Nonlinear interactions: time-averaged inhibition}

We addressed the question of neural interactions within the population representation. If there were no interactions within the population, then the DPAs of the composite stimuli would be predicted to be the linear superpositions of the DPAs of the component elementary stimuli. To test this null hypothesis, we build DPAs based on the same estimator used for elementary stimuli, but now weighting the contribution of each cell with the firing rate observed in response to the composite stimuli.

First, we examined interaction effects by comparing the timeaveraged (from 30 to $80 \mathrm{msec}$ ) population representations. Figure 6 illustrates the DPAs derived by interpolation; Figure 7 the OLE-derived DPAs of composite stimuli and their superpositions. Both the measured and the superimposed DPAs are monomodal for small, and bimodal for large stimulus separations, the transition occurring at $\sim 1.6^{\circ}$ separation.

The most striking deviation from the linear superposition (Fig. 6, bottom; Fig. 7, dashed line) was a reduction of activity compared to the measured responses (Fig. 6, top; Fig. 7, solid line), which is particularly strong for small stimulus separations. This reduction is not caused by a saturation of population activity because it is also observed for composite stimuli of larger separations where the distributions are bimodal and have little overlap. Note that in this case the levels of activation in the composite representations are even lower than for the corresponding elementary stimuli (see Fig. 9B, horizontal arrow). A quantitative assessment of this inhibitory interaction allows to uncover its dependence on stimulus distance. The total activation in the population distribution was computed as the area under the distribution and is expressed as a percentage of the total activation contained in the superposition. This percentage is always $<100 \%$, indicating inhibition, which is strongest for small distances and decreases with increasing distances (Fig. 8).

A slight gradient of the amplitudes and the time courses within the DPAs of the elementary stimuli was assumed to account for the asymmetric deviations of the measured distributions compared to the superpositions at 1.2 and $1.6^{\circ}$ stimulus separation (Fig. 7). Therefore, interaction processes may amplify this inhomogeneity by shifting the maximal amplitude of the distributions toward the nasally located stimulus component (for details, see "Dynamic neural field model"). Note that the inhomogeneity became additionally apparent in the superpositions of the Gaussian-interpolated DPAs (Fig. 6). In contrast to the optimal estimation procedure, this method does not normalize the small gradient of amplitudes observed in the distributions of the elementary stimuli.

\section{Nonlinear interaction: early excitation-late inhibition}

To investigate the time structure of interaction, we further analyzed the OLE-derived DPAs by comparing representations of composite stimuli either to the representations of elementary stimuli or to their superpositions. We therefore calculated the activation around the nasally positioned component because it was at the same retinal location for all composite stimuli. As a quantitative measure, we integrated activity within a band of $\pm 0.4^{\circ}$ around that particular visual field position (Fig. $9 A$, vertical arrow).

Figure $9 B$ (solid line) displays the temporal evolution of activity at $5 \mathrm{msec}$ intervals for the different composite stimuli (illustrated in Fig. 9A). The response to the nasally positioned elementary stimulus alone is shown as a dashed line. There are notable differences between elementary and composite stimuli in an early and a late response epoch. At small separations between the component stimuli, the response has a $7 \mathrm{msec}$ shorter latency $(p<0.001$, ANOVA) as compared to the single stimulus condition. This is accompanied by an earlier onset of the decay of the population activity. By contrast, the late part of the response is always inhibited.

For quantitative evaluation, we divided time into an early 


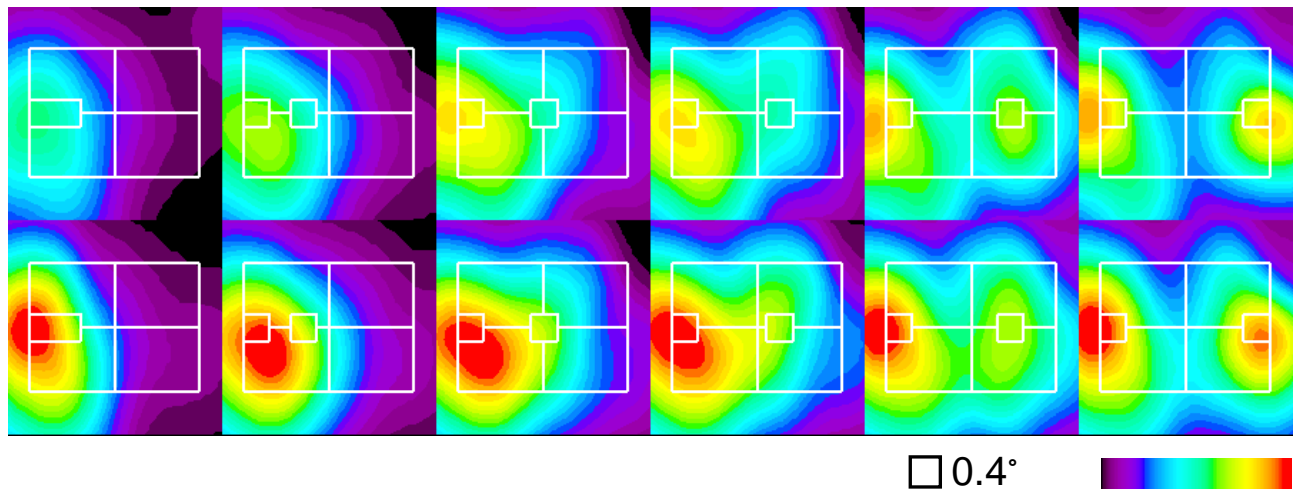

Figure 6. The measured two-dimensional DPAs (top) of composite stimuli (from left to right, $0.4-2.4^{\circ}$ separation) were compared to the superpositions of the representations of their component elementary stimuli (bottom). The DPAs were based on spike activity of 178 cells averaged over the time interval from 30 to $80 \mathrm{msec}$ after stimulus onset. Same conventions as in Figure $2 B$, the color scale was normalized to peak activation separately for each column. For small stimulus separation, note the remarkably reduced level of activation for the measured as compared to the superimposed responses. The bimodal distribution recorded for the largest stimulus separation comes close to match the superposition. However, inhibitory interaction can still be observed.
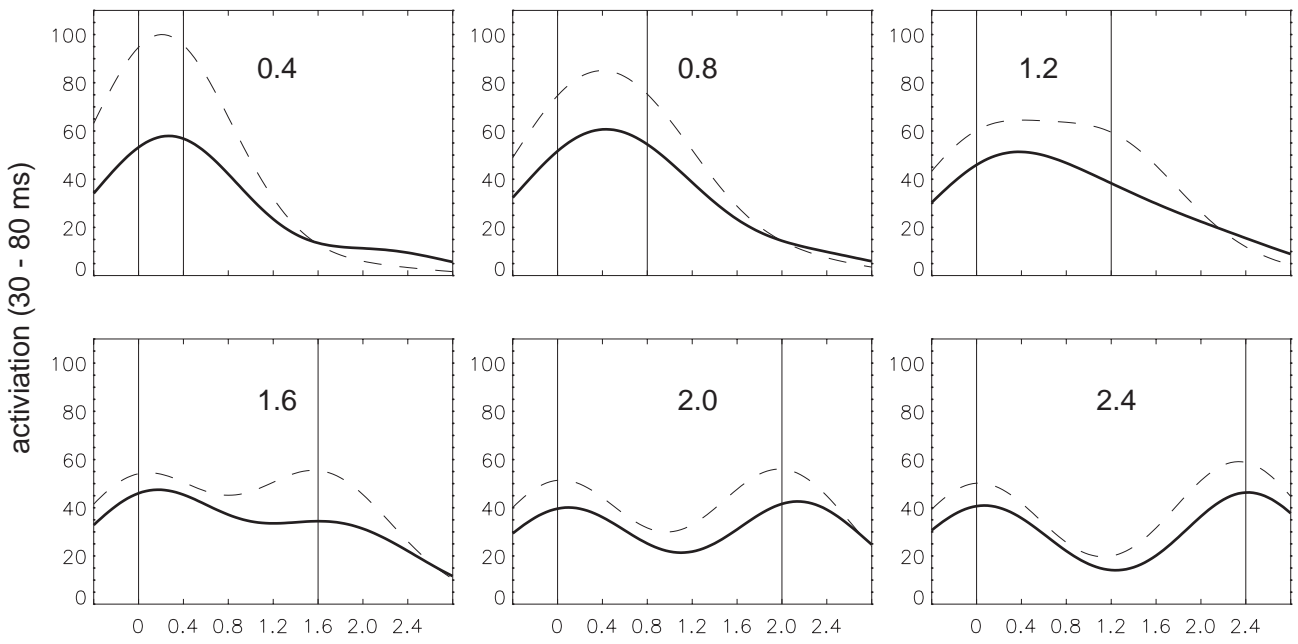

[deg]

Figure 7. The OLE-derived DPAs for the composite stimuli as depicted in Figure 6. Solid lines mark the measured activations, and dashed lines show the calculated superpositions (vertical lines mark stimulus positions). Peak activation was uniformly normalized. As demonstrated for the interpolated two-dimensional DPAs, the level of measured activation was systematically reduced for smaller stimulus separations but approached linear superposition for larger separations. The transition from monomodal to bimodal distributions was found between 1.2 and $1.6^{\circ}$ separation. A slight asymmetry of the amplitudes between the representations of the left and the right stimulus component was found for the measured as compared to the superimposed distributions for stimulus separations of 1.2 and $1.6^{\circ}$.

$(30-45 \mathrm{msec})$ and a late $(45-80 \mathrm{msec})$ epoch. For the early period, we compared the population representation of composite stimuli to the superpositions. Because we expect to find excitatory interaction, this is a conservative comparison, because saturation effects would tend to limit the responses. The solid line in Figure 10 shows the difference between the activation in response to the composite stimuli and the activation in the superimposed responses expressed in percent of the latter. In this early response epoch, there was more activation in the measured than in the superimposed responses at all distances except the largest $\left(2.4^{\circ}\right)$. This excess activation, which reached a maximum of $58 \%$ at a stimulus distance of $1.6^{\circ}$, is evidence of distance-dependent excitatory interaction during the build-up phase of the DPAs of composite stimuli.

That the activation with composite stimuli exceeded even that of the superpositions demonstrates that response saturation is not the cause of the apparent inhibitory interactions observed in the time-averaged analysis. Accordingly, the time-averaged inhibitory effect (compare Figs. 6, 7) originates from the late response epoch of 45-80 msec after stimulus onset. For this epoch, the dashed line in Figure 10 shows the relative difference of responses to composite as compared to elementary stimuli. At all stimulus separations, the difference is negative, indicating inhibition below the activation level for a single stimulus. This inhibition is slightly stronger for larger stimulus separations, providing further evidence for distance-dependent late inhibitory interaction. Moreover, it confirms that response saturation is not an explanation for this inhibitory effect.

\section{Spatial interaction: repulsion effect}

The neural field model predicts (see next section) that inhibitory interactions are dominant at larger distances, resulting in a repulsion effect for the apparent position of two stimulus components. We tested this prediction using the OLE-derived distribu- 


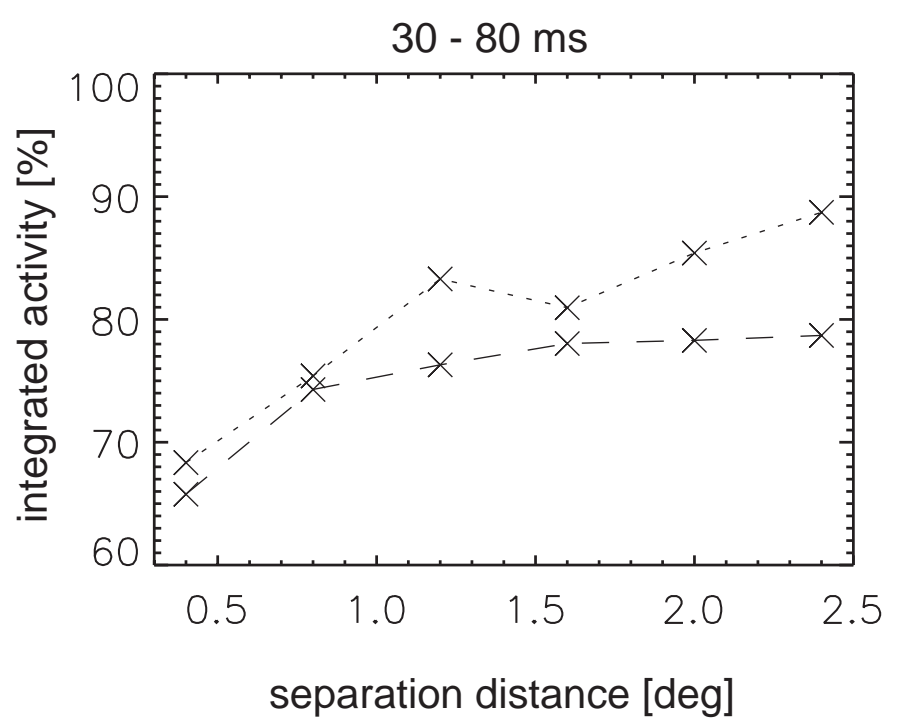

Figure 8. Reduction of the DPA magnitude induced by composite stimuli as a function of separation between the two component stimuli, calculated for the time-averaged responses $(30-80 \mathrm{msec})$. The total activation in the distribution was expressed as percentage of the total activation in the superposition. The dashed line marks results from the OLEderived DPAs, the dotted line depicts results from the two-dimensional distributions (Gaussian interpolation). For both ways of construction, inhibition was strongest for zero distance (66\% for the OLE-derived, 68\% for the interpolated data) and decreased almost monotonically with increasing distance, but was still present at the largest separation tested $\left(2.4^{\circ}\right)$.

tions. As described, the DPAs were bimodal at stimulus separations between 1.6 and $2.4^{\circ}$. In fact, at these distances we found that the maxima of the DPAs were shifted outward by $\sim 0.3^{\circ}$ as compared to the corresponding maxima of the superposition (Fig. 11). This repulsion effect was particularly strong in the time window of 60-80 msec after stimulus onset, where inhibition is dominant.

Note that all results concerning interaction and temporal evolution were equivalent when obtained from the two different approaches of DPA construction.

\section{Dynamic neural field model}

A theoretical model of the temporal evolution of the population representation and the interaction effects is formulated to substantiate our theoretical interpretation of the results. The model is embedded in a general framework that bridges neuronal and behavioral levels of description (for review, see Schöner et al., 1997). The elementary stimuli flashed at different positions on a horizontal line in the visual field are thought of as defining a one-dimensional space, in which the dependence of interaction on distance is probed. At each position, $x$, an activation variable, $U(x)$, is introduced that defines a field of neural activation along the horizontal dimension of visual space.

This neural field is assumed to evolve continuously in time under two different types of inputs: (1) afferent input from sensory stimulation activates those regions of the field that represent the specified values of the parameter space; and (2) inputs from interaction processes within the field exert excitatory or inhibitory effects onto the field. What locations excite or inhibit each other is determined by interaction kernels $w_{\mathrm{u}}(x)$ and $w_{\mathrm{v}}(x)$, respectively. These are derived under the assumption that nature and strength of the interactions between different sites in the field depend on the distance between those sites. The identification of appropriate kernels, which can explain the overall time scale of build-up and decay as well as the spatial width of the measured population responses, is thus the primary modeling task. The modeling is not aimed to reproduce the experimental data in all detail, but to identify a simple mathematical description that can be used to support and clarify the interpretation of the main experimental findings.

As a rule, the response of the neural population to briefly flashed visual stimuli is transient. The time structure of the DPAs reveals dynamic properties of the cortical neural network that go beyond passive filtering. We refer to such responses as active or self-generated transients. To account for this nontrivial time structure of the population response, we introduce a second variable at each site of the field. This variable is excited by activation in the $u$ field and inhibits, in turn, that field at the corresponding site.

The mathematical description we use is:

$$
\begin{aligned}
\tau \dot{u}(x, t)= & -u(x, t)+h+S(x, t)+\cdots \\
& +F(u(x, t))\left[\int w_{u}\left(x-x^{\prime}\right) F\left(u\left(x^{\prime}, t\right)\right) d x^{\prime}-v(x, t)\right] \\
\tau \dot{v}(x, t)= & -v(x, t)+\int w_{v}\left(x-x^{\prime}\right) F\left(u\left(x^{\prime}, t\right) d x^{\prime}\right.
\end{aligned}
$$

A similar mathematical framework has been used by Amari (1977) to discuss the dynamics of pattern formation in cortical neuronal tissues. He focused primarily on stable stationary states, consisting of localized peaks of activation, whereas only spatially homogeneous (nonlocal) patterns were studied as transient solutions.

The lateral connections are functions of the distance $\left(x-x^{\prime}\right)$ of positions $x, x^{\prime}$ in visual space. Numerical studies with different types of kernels (e.g., Gaussians, exponentials, rectangular forms) revealed that the interesting qualitative properties of the solutions of Equation 9 are largely independent of the particular analytical form of the kernels $w_{\mathrm{u}}$ and $w_{\mathrm{v}}$, as long as they preserve characteristic relationships of amplitude of inhibition and excitation as well as of the spatial range of these two factors. The simulations shown below are based on two Gaussians:

$w_{u}\left(x, x^{\prime}\right)=\mathrm{A}_{u} \exp \left[-\frac{\left(x-x^{\prime}\right)^{2}}{2 \sigma_{u}^{2}}\right]$
$w_{v}\left(x, x^{\prime}\right)=\mathrm{A}_{v} \exp \left[-\frac{\left(x-x^{\prime}\right)^{2}}{2 \sigma_{v}^{2}}\right]$

where the amplitudes $\mathrm{A}_{\mathrm{u}}, \mathrm{A}_{\mathrm{v}}$ and the range parameters $\sigma_{\mathrm{u}}, \sigma_{\mathrm{v}}$ are positive constants. A general constraint arises from the requirement that the excitatory response does not spread out. This imposes that the spatial range of inhibitory interactions must exceed that of excitatory interactions $\left(\sigma_{\mathrm{v}}>\sigma_{\mathrm{u}}\right)$.

The threshold function $F$ in Equation 9 must be monotonically increasing and nonlinear, but again its particular functional form is of little importance for the qualitative behavior of the field dynamics. We used the well-known sigmoidal function $F(u)=$ $1 /[1+\exp (-b u)]$. For given interaction kernels, a lower limit for the slope $b>0$ can be obtained such that the existence of self-generated, transient responses is guaranteed.

The interaction terms are multiplied by the state-dependent 
A
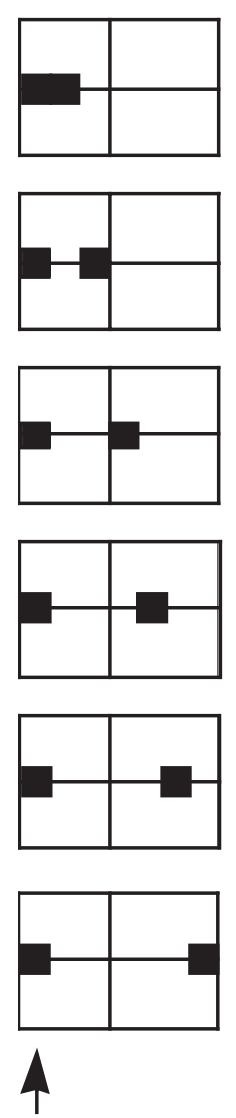

B
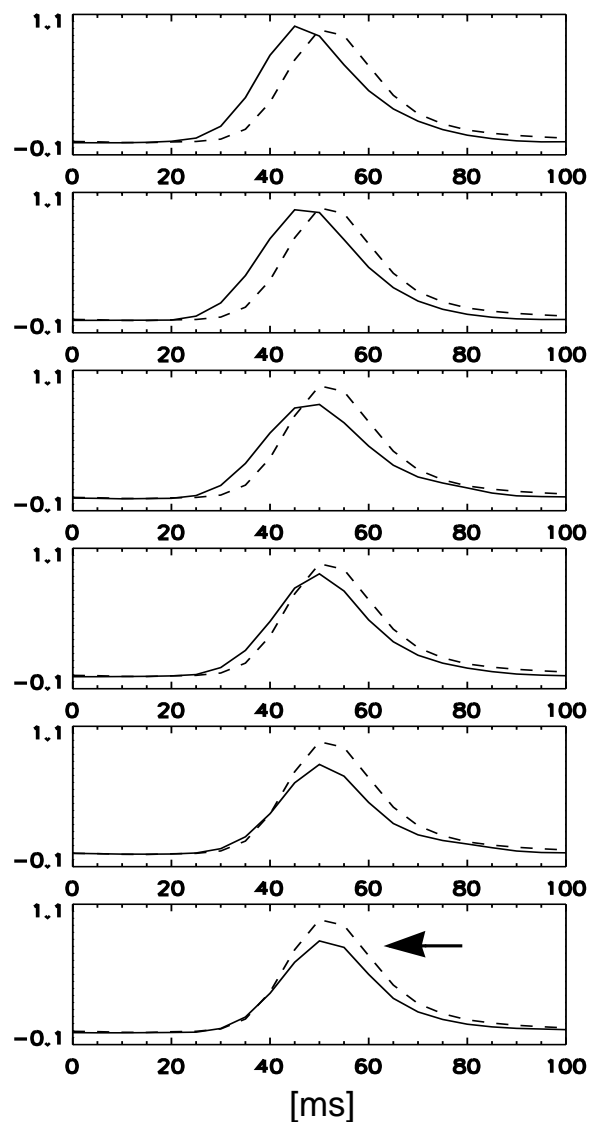

C
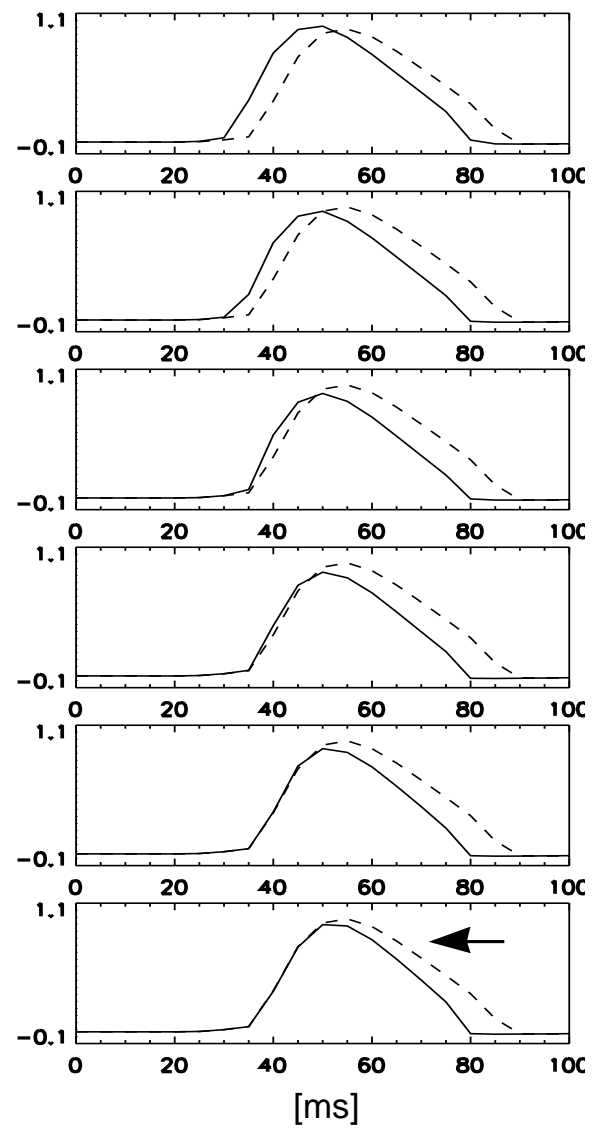

Figure 9. Time-resolved analysis of interaction effects based on integrals of DPAs in a $0.8^{\circ}$ wide band around the location of the nasally positioned elementary stimulus $(A$, vertical arrow). The different composite stimuli are shown in column $A$. Column $B$ contrasts the OLE-derived DPAs to composite stimuli (solid line) with the responses to the single nasally positioned elementary stimulus (dashed line). At small distances, the activation to composite stimuli had a significantly smaller latency accompanied by an earlier onset of the decay of the population activity as compared to the elementary stimuli. The late part of the responses to the composite stimuli was characterized by an overall inhibition. The arrow marks that peak activation in response to the composite stimulus of largest separation is still below activity measured in the single stimulus condition. Column $C$ displays results of simulations of the dynamic neural field model scaled to match the experimental stimulus conditions. Parameter values used for this simulation are: $A_{\mathrm{u}}=5.2, A_{\mathrm{v}}=$ $4, \sigma_{\mathrm{u}}=15, \sigma_{\mathrm{v}}=25, A_{\mathrm{s}}=4, B_{\mathrm{s}}=10, b=1, h=-3, \tau=15$. The arrow marks that inhibition can still be seen at the largest probed distances between the component stimuli.

sigmoidal signal $F(u)$. This factor prevents the asymptotic transient response to fall below resting level because only those sites in the field that are sufficiently activated are susceptible to inhibitory interaction.

The parameter $\tau$, Equation 9, determines the overall time scale of build-up and decay of the field activity and can be adjusted to reproduce qualitatively the measured time course of population activity changes. In the numerical studies, we have used the value $\tau=15$. A fixed criterion (5\% above resting level) was used to define the response onset in the experiments. For the simulations, the afferent transient stimulus $S(x, t)$ at position $x$, applied for a duration $\Delta t=25 \mathrm{msec}$, is a Gaussian profile characterized by its strength, $A_{\mathrm{s}}$, and width parameter, $2 \sigma$. The choice of $\sigma$ fixes the spatial units relative to the experimental space scale. All range parameters used in the model simulations were chosen as multiples of $\sigma=5$, which represents $0.2^{\circ}$ in visual space.

If this transient external input creates enough excitation within the field, the excitatory response develops a single spatial maximum located at the center, $x$, of the stimulated segment. This is followed by a process of relaxation to the resting state driven by increasing inhibition in the field. The activation level of this resting state is a homogenous and stable solution of the model dynamics, fixed by the parameter $\mathrm{h}<0$ ( $\mathrm{h}=-3$ for the simulations shown here).

\section{Simulation results}

Figure 9 compares the temporal evolution of population activation in the experiment (B) and in the model (C). Composite stimuli with six spatial separations were used. The same normalization procedures for the simulated data were applied as for the experimental data. To further facilitate the comparison of theory and experiment, a time interval of $25 \mathrm{msec}$ before stimulus onset was added, so that the field dynamics has relaxed to its resting state. This time window accounts for the temporal delay between the stimulus presentation and the cortical response in the experiment.

Distance-dependent early excitation and late inhibition are observed by comparing the temporal evolution of the field in response to the single input at the nasal location. Note that in the experiment, the limit case of two independent peaks not interacting at all is not reached even at the largest probed distances between the component stimuli. At that largest separation, an 


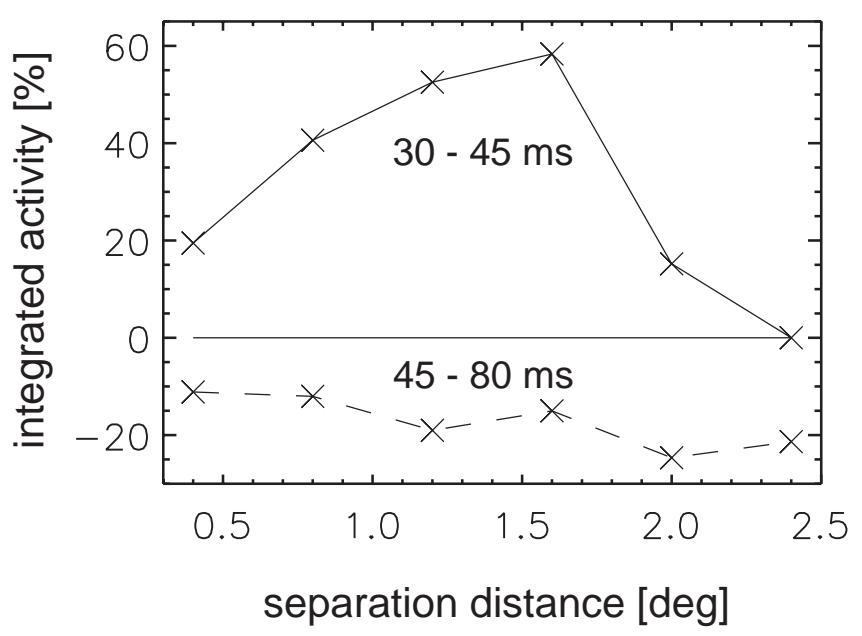

Figure 10. Interaction effects analyzed in two separate time intervals. The OLE-derived DPAs were integrated within a band of $0.8^{\circ}$ width around the nasally positioned stimulus component. For the early response epoch, between 30 and $45 \mathrm{msec}$ after stimulus onset, we computed the relative difference between the activation in the distribution of the composite stimulus and the superposition. This difference (solid line) is shown as a function of the spatial separation of the two stimulus components. Note that the positive values peaking at $1.6^{\circ}$ with $58 \%$ enhancement remain positive up to $2.0^{\circ}$. This excess activation is indicative of excitatory interaction in this early response time interval. For the response epoch between 45 and $80 \mathrm{msec}$, we computed the relative difference between the activation in the distribution of composite stimuli, and the activation in response to the single nasally positioned elementary stimulus (dashed line). For all separations, this difference was negative, reaching $\sim 25 \%$ inhibition at $2.0^{\circ}$, indicative for inhibitory interaction. The distinct inhibition still present at the largest separation makes it unlikely that a local saturation effect can explain this observation. For the two epochs, the different types of analysis used provide a conservative estimate: for excitatory interaction in the first case, for inhibitory interaction in the second case (for details, see Results).

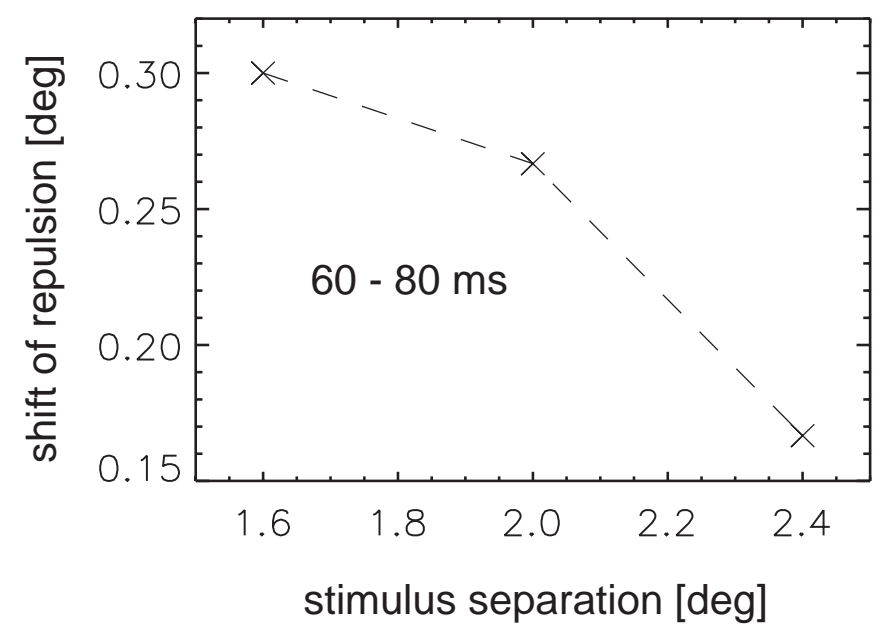

Figure 11. Spatial repulsion effect. Repulsion was computed in the time slices between 60 and $80 \mathrm{msec}$ after stimulus onset where inhibition is dominant. The distances between the activity peaks in the bimodal DPAs (OLE-derived) for stimulus separations of $1.6-2.4^{\circ}$ were compared to the respective superpositions. Differences were depicted as shifts of repulsion dependent on stimulus separation. Maximal spatial shift amounts to $0.3^{\circ}$.

inhibition effect can still be seen in the time course of activation (Fig. 9B, C, horizontal arrows).

In the spatial domain, nonlinear interactions are observed as
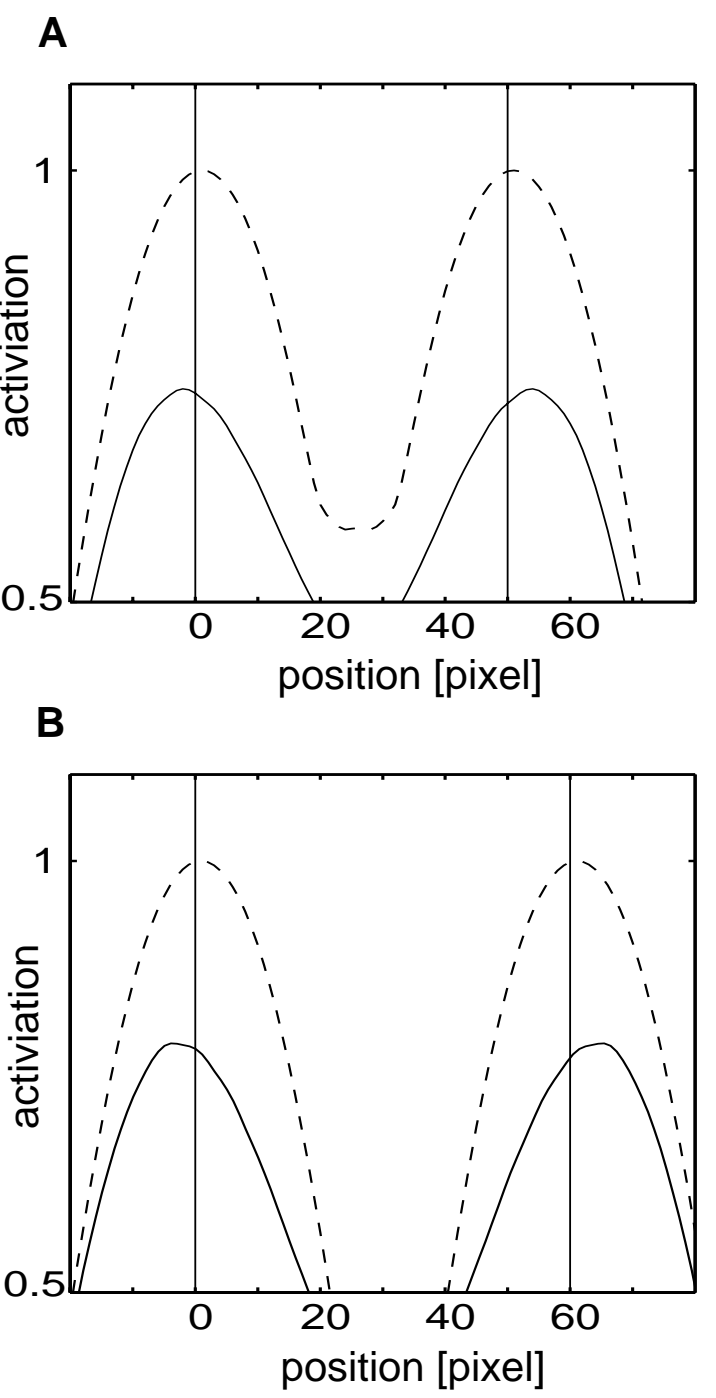

Figure 12. Simulation of the repulsion effect. Shown are the simulated DPAs in response to the composite stimuli of $2.0^{\circ}(A)$ and $2.4^{\circ}(B)$ separation (solid lines). These are compared to the superpositions (dashed lines). The two vertical lines mark the position of the elementary stimuli. The same values of the model parameters were used as in Figure $9 C$. Repulsion is manifested by an outward shift of the maxima.

differences in shape and location of the time-averaged spatial profile (Fig. 12A,B) of the calculated superposition compared to the composite stimulation. In the model, the two excited regions attract each other to unite into one excited region when they interact directly through the excitatory connections. Conversely, when two peaks of activation are induced at somewhat larger distances, they interact primarily through the longer range inhibitory interactions, and this leads to the documented repulsion of the two peaks.

To further emphasize the role of time in the interaction process, we have explored the influence of a small inhomogeneity (up to $5 \mathrm{msec}$ ) in the temporal evolution of the field on the emerging activity patterns. A slightly faster growth of activation at one field site causes an asymmetry in the competition strength between neighboring activity peaks. In each time step, the activitydependent strength of inhibition exerted by the other local excitation is always smaller for the temporally privileged location. This imbalance finally leads to a difference in peak amplitude at 


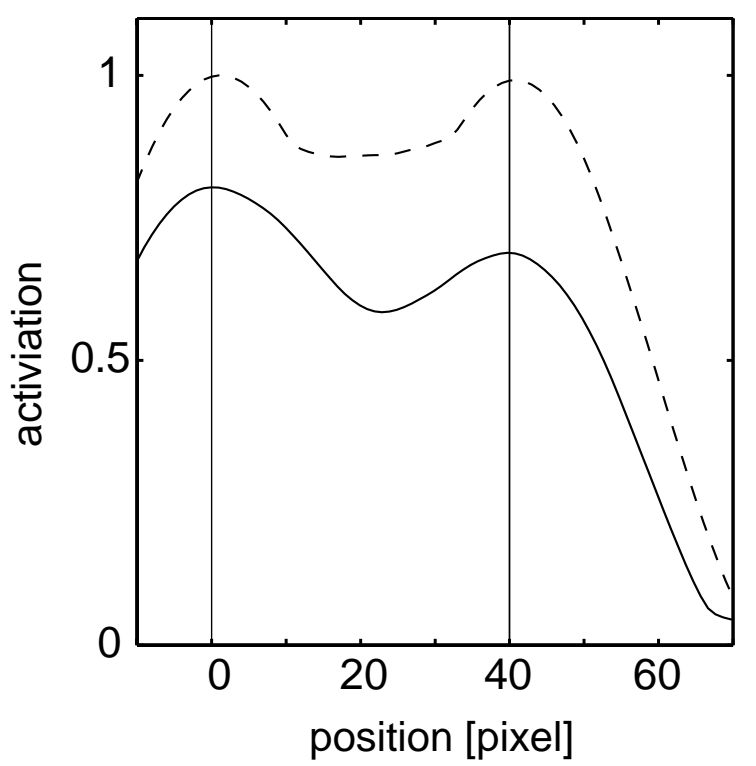

Figure 13. Effect of a nonhomogeneous, position-dependent evolution of field activity. The time-averaged response period of the superposition (dashed line) and the response to composite stimuli (solid line) are compared for a separation of $1.6^{\circ}$. A reduction of input strength (30\% in the simulation shown here) for the right stimulus leads to a delay in temporal evolution at this particular field site. Because this reduction has little effect on the response maximum in the single stimulus condition, the timeaveraged superposition profile is still symmetric. As a result of field interactions, a significant decrease of activity at the right position occurs when the two stimuli are applied together.

the two stimulation sites. Note, however, that the averaged superposition profile can still be symmetric when the mechanism that causes the difference in the temporal evolution has little effect on the maximum peak response of the elementary stimuli. This condition can easily be met by introducing a position-dependent slight variation of the input strength (compare Figs. 7, 13).

\section{DISCUSSION}

Effects of interaction across distributed cortical representations are widely discussed as an important aspect of cortical function. If interaction contributes significantly to neural activation in visual cortex, then representations of the visual environment will differ from a simple feedforward remapping of visual space. To investigate the presence and magnitude of interaction processes in cat primary visual cortex, we constructed DPAs from the activity of an ensemble of neurons in response to single squares of light.

\section{Construction of distributions of population activation}

Using two different approaches, DPAs were defined in parameter space of the visual field which enabled us to analyze ranges of excitatory and inhibitory interactions in terms of the stimulus metrics.

Instead of asking how accurately the parameter of stimulus location can be reconstructed or decoded, we primarily were interested in analyzing interaction-based deviations of population representations dependent on defined variations of stimulus configurations. Accordingly, there is an important point of departure from the interest we share with aspects relating to estimation theory. Our analysis aimed to investigate how the representation of retinal position evolves in time and how it is affected by interaction among neurons. Besides, reading out discrete sample points such as peak maxima does not imply that the brain actually uses such measures for decoding.

When composite stimuli consisting of two squares of light were used, the deviations of the distributions from additivity were considered as active contributions from neural interaction, i.e., how interactions distort the distribution of activation. We conclude that such contributions can be regarded as additional information generated by the neural system dependent on context and its actual state.

It is important to note that both approaches used to derive DPAs revealed qualitatively equivalent results, implying that the exact way of how the distributions were constructed was not crucial for the observed interaction effects.

\section{Interaction within the population representation of composite stimuli}

The use of time-resolved DPAs allowed us to identify signatures of interaction processes that were dependent on time and on the distance of the composite stimuli. In the first 30-45 msec after stimulus onset we found evidence for excitatory interaction, which decreases with increasing distance between the two components. In contrast, when activation was integrated over the later part of the response (45-80 msec after stimulus onset), inhibitory interaction dominated. We provided several arguments that exclude saturation of neural firing rates as an alternative explanation.

An additional indication for the presence of inhibitory interaction was found by analyzing the spatial shapes of the DPAs. Mutual repulsion of the maxima of the DPAs was observed at stimulus separations between 1.6 and $2.4^{\circ}$, at which the distributions were bimodal. Such repulsion effects qualitatively match psychophysical results obtained from humans. Errors incurring when human subjects estimate the visual distance between two spots of light depend systematically on the retinal distance of the stimuli. Small separations are underestimated, large distances are overestimated (Hock and Eastman, 1995). Similar results have been obtained for estimation of the orientation of stimuli (Westheimer, 1990; for theoretical modeling see Lehky and Sejnowski, 1990). In addition, mislocation effects have been described for other sensory modalities, such as the tactile saltation effect (Geldard and Sherrick, 1972), supporting the assumption of a general cortical nature of such phenomena (Kalt et al., 1996).

\section{Dynamic neural field model}

A dynamic neural field model was introduced for theoretical treatment of the dynamics of neural population activity (Schöner et al., 1997). Models of the same mathematical format have been proposed in the past as models of dynamic cortical processing (Wilson and Cowan, 1973; Amari, 1977). It is important to note that the entire set of experimental results could be accounted for from a single set of parameter values. The construction of the population representation was used to map neural data onto the visual field. Correspondingly, the neural field was likewise defined over visual space. The activation variables $u$ and $v$ in Equation 9 represent the accumulated excitation and inhibition within the population of neurons. The structure of the postulated interaction function consists of both excitatory and inhibitory coupling. Because the amplitude of the excitatory contribution to interaction is higher and its spatial extent is narrower than for the inhibitory contribution, the net interaction within the representation is excitatory over short distances in visual space, and inhibitory at larger spatial separation. 
The absolute values of range parameters used for the numerical studies revealed that the excitatory and the inhibitory processes extend over a range of 0.6 and $1.0^{\circ}$ of visual field, respectively. The strength of inhibition and excitation strongly influences the width of the emerging activity distribution, and thus the spatial separation at which a transition from a monomodal to a bimodal representation occurs. Our simulations showed that even those representations that overlap only for the smallest separation still can reveal the effect of late inhibition and early excitation, indicating that the width of the distributions has only little effects on the time course of interaction. A small number of parameters were sufficient for modeling the complex spatiotemporal responses from many different cell types combined at a population level.

\section{Relationship of our results to single cell analysis}

Interaction profiles have been repeatedly examined at the level of single cells (Movshon et al., 1978; Heggelund, 1981a,b; Nelson, 1991; Tolhurst and Heeger, 1997). In those studies, the activity of a cell induced by a single stimulus at the RF center was compared to the activity of the cell in the presence of a second stimulus presented at varied locations.

In contrast, the population approach used here performs two different types of averages. First, because our stimuli were not RF-centered, we average across different spatial locations within the RFs (cf. Szulborski and Palmer, 1990). Outside the laboratory, visual objects are similarly distributed in arbitrary ways across RFs, so that this way of stimulus presentation and averaging is crucial for an understanding of how complex scenes are represented in visual cortex.

Second, we average across many different cell types. Neurons in area 17 contribute potentially to the representations of many different parameters such as retinal position, orientation, curvature, length, motion direction, etc. To characterize the contribution of each neuron to the representation of stimulus location, one might conceive of the high-dimensional space spanned by these different parameters. Each neuron could be thought of as a point in this parametric space. This point corresponds to a set of preferred values for all represented parameters. By asking only how the firing rate of the neuron depends on visual field position, the contributions of all neurons are averaged, although their preferred parameter set may be different along other dimensions. In this sense, the DPA is a projection from a potentially highdimensional space onto a common neuronal space representing only visual field position. The DPA could thus be viewed as a neural population receptive field of the inverted cortical pointspread function ("cortical spread-point function").

\section{The shape of the DPA matters}

Population coding ideas have largely been centered on estimating the stimulus or task parameter from the activity of populations of neurons (Georgopoulos et al., 1986, 1993; Vogels, 1990; Zohary, 1992; Seung and Sompolinsky, 1993; Salinas and Abbott, 1994; Groh et al., 1997). Compared to vector-based population techniques, the current approach focused on the concept of an entire distribution of population activation (Lee et al., 1988; Bastian et al., 1997; Pouget et al., 1998) (for related attempts, see Anderson, 1994a,b; Zemel et al., 1998, in which they seek to recover a probability distribution of activity over the encoded variable). In our approach, the distribution is significant not only by a mean value of the represented parameter, but also through its shape. Consequently, asymmetric deformations of the DPA could be detected, in which two peaks in the DPA are repelled from each other at sufficiently large stimulus separations. This effect is observable only by taking the shape of the constructed DPA into account and would be detectable neither on the basis of PSTH responses of individual cells nor in reconstructions that estimate only single values or discrete samples of parameters.

\section{Relationship of our results to cortical maps}

In principle, our time averaged two-dimensional DPAs are equivalent to activities recorded in functional imaging studies such as functional magnetic resonance imagine, positron emission tomography, and optical imaging of intrinsic signals assuming a clean retinotopy. There are a number of differences, however. Besides the limitations of these techniques to resolve the millisecond time scale as accomplished by our single cell recordings, the main problem arises from the fact that the retinotopy is far from coming close to a clean representation of the visual field (cf. Das and Gilbert, 1997). This is particularly obvious at the spatial scale of our investigation, which differentiates between visual angles $<1^{\circ}$ apart (Hubel and Wiesel, 1962; Albus, 1975). Analysis of the cortical point-spread function has shown that the processing of even very small stimuli is associated with a widespread pattern of cortical activation (Grinvald et al., 1994; Godde et al., 1995; Chen-Bee and Frostig, 1996). In addition, imaging methods as listed above do not solely reflect spike activity but include contributions from glial cells and cerebral blood flow. Accordingly, comparison of DPAs spanned in parametric space with cortical activation maps recorded with such imaging techniques may allow separating neural and non-neural contributions.

A dynamically distributed processing over a large cortical area possibly reflects a major role in neural strategies of cooperative interaction. Observations in real-time imaging studies supported this assumption because the firing of single neurons can be predicted if the whole pattern of cortical population activation is taken into account (Arieli et al., 1996; Kenet et al., 1998). Because our approach allows for a functional interpretation of cortical activation patterns, it may serve to find transformation rules that map the multidimensional visual input onto cortical representations.

\section{REFERENCES}

Albus K (1975) A quantitative study of the projection area of the central and the paracentral visual field in area 17 of the cat. I. The precision of the topography. Exp Brain Res 24:159-179.

Allman J, Miezin F, McGuiness EL (1985) Stimulus specific responses from beyond the classical receptive field. Annu Rev Neurosci $8: 407-430$

Amari S (1977) Dynamics of pattern formation in lateral-inhibition type neural fields. Biol Cybern 27:77-87.

Anderson CH (1994a) Basic elements of biological computational systems. Int J Modern Physics C 5:135-137.

Anderson CH (1994b) Neurobiological computational systems. In: Computional intelligence imitating life (Palaniswami M, Attkiouzel Y, Marks II RJ, Fogel D, Fukada T, eds), pp 219-222. New York: IEEE.

Arieli A, Sterkin A, Grinvald A, Aertsen A (1996) Dynamics of ongoing activity: explanation of the large variability in evoked cortical responses. Science 273:1868-1871.

Bastian A, Riehle A, Erlhagen W, Schöner G (1997) Prior information preshapes the population representation of movement direction in motor cortex. NeuroReport 9:315-319.

Bringuier V, Chavane F, Glaeser L, Fregnac Y (1999) Horizontal propagation of visual activity in the synaptic integration field of area 17 neurons. Science 283:695-699.

Chen-Bee CH, Frostig RD (1996) Variability and interhemispheric asymmetry of single-whisker functional representations in rat barrel cortex. J Neurophysiol 76:884-894.

Creutzfeldt OD, Garey LJ, Kuroda R, Wolff JR (1977) The distribution 
of degenerating axons after small lesions in the intact and isolated visual cortex of the cat. Exp Brain Res 27:419-440.

Das A, Gilbert CD (1997) Distortions of visuotopic map match orientation singularities in primary visual cortex. Nature 387:594-598.

Dinse HR (1986) Foreground-background-interaction - Stimulus dependent properties of the cat's area 17,18 and 19 neurons outside the classical receptive field. Perception 15:A6.

Dinse HR, Krüger K, Best J (1990) A temporal structure of cortical information processing. Concept Neurosci 1:199-238.

Erickson RP (1974) Parallel population coding in feature extraction. In: The neurosciences (Schmitt FO, Worden FG, eds), pp 155-169. Cambridge: MIT.

Fisken RA, Garey CJ, Powell TPS (1975) The intrinsic, association and commissural connections of area 17 of the visual cortex. Philos Trans R Soc Lond B Biol Sci 272:487-536.

Gaal G (1993a) Population coding by simultaneous activities of neurons in intrinsic coordinate systems defined by their RF weighting functions. Neural Networks 6:499-515.

Gaal G (1993b) Calculation of movement direction from firing activities of neurons in intrinsic coordinate systems defined by their preferred directions. J Theor Biol 162:103-130.

Geldard FA, Sherrick CE (1972) The cutaneous "rabbit": a perceptual illusion. Science 178:178-179.

Georgopoulos AP, Schwartz AB, Kettner RE (1986) Neural population coding of movement direction. Science 233:1416-1419.

Georgopoulos AP, Taira M, Lukashin A (1993) Cognitive neurophysiology of the motor cortex. Science 260:47-52.

Gielen CCAM, Hesselmans GHFM, Johannesma PIM (1988) Sensory interpretation of neural activity patterns. Math Biosci 88:14-35.

Giese MA, Cubaleska B, Pagel M, Akhavan AC, Jancke D, Dinse HR, Schöner G (1997) Identification of feedforward and recurrent part of the neural dynamics of position representation in cat area 17 . Soc Neurosci Abstr 23:2364.

Gilbert CD, Wiesel TN (1979) Morphology and intracortical projections of functionally identified neurons in cat visual cortex. Nature 280:120-125.

Gilbert CD, Wiesel TN (1990) The influence of contextual stimuli on the orientation selectivity of cells in primary visual cortex of the cat. Vision Res 30:1689-1701.

Godde B, Hilger T, von Seelen W, Berkefeld T, Dinse HR (1995) Optical imaging of rat somatosensory cortex reveals representational overlap as topographic principle. NeuroReport 7:24-28.

Grinvald A, Lieke EE, Frostig RD, Hildesheim R (1994) Cortical pointspread function and long-range lateral interactions revealed by realtime optical imaging of macaque monkey primary visual cortex. J Neurosci 14:2545-2568.

Groh JM, Born RT, Newsome WT (1997) How is a sensory map read out? Effects of microstimulation in visual area MT on saccades and smooth pursuit eye movements. J Neurosci 17:4312-4330.

Heggelund P (1981a) RF organization of simple cells in cat striate cortex. Exp Brain Res 42:89-98.

Heggelund P (1981b) RF organization of complex cells in cat striate cortex. Exp Brain Res 42:99-107.

Hock HS, Eastman KE (1995) Context effects on perceived position: sustained and transient temporal influences on spatial interactions. Vision Res 35:635-646.

Hubel DH, Wiesel TN (1962) Receptive fields, binocular interaction and functional architecture in the cat's visual cortex. J Physiol (Lond) 160:106-154.

Jancke D, Akhavan AC, Erlhagen W, Schöner G, Dinse HR (1996) Reconstruction of motion trajectories using population representations of neurons in cat visual cortex. Soc Neurosci Abstr 22:646.

Kalt T, Akhavan AC, Jancke D, Dinse HR (1996) Dynamic population coding in rat somatosensory cortex. Soc Neurosci Abstr 22:105.

Kenet T, Arieli A, Grinvald A, Shoham D, Pawelzik K, Tsodyks M (1998) Spontaneous and evoked firing of single cortical neurons are predicted by population activity. Soc Neurosci Abstr 24:1138.

Kisvárday ZF, Eysel UT (1993) Functional and structural topography of horizontal inhibitory connections in cat visual cortex. Eur J Neurosci 5:1558-1572.
Lee C, Rohrer WH, Sparks DL (1988) Population coding of saccadic eye movements by neurons in the superior colliculus. Nature 332:357-360.

Lehky SR, Sejnowski TJ (1990) Neural model of stereoacuity and depth interpolation based on a distributed representation of stereo disparity. J Neurosci 10:2281-2299.

Movshon JA, Thompson ID, Tolhurst DJ (1978) RF organization of complex cells in the cat's striate cortex. J Physiol (Lond) 283:79-99.

Müsseler J, Van der Heijden AHC, Mahmud SH, Deubel H, Ertsey S (1999) Relative mislocalization of briefly presented stimuli in the retinal periphery. Percept Psychophys, in press.

Nelson SB (1991) Temporal interactions in the cat visual system. I. Orientation-selective suppression in the visual cortex. J Neurosci 11:344-356.

Nicolelis MAL, Chapin JK (1994) Spatiotemporal structure of somatosensory responses of many-neuron ensembles in the rat ventral posterior medial nucleus of the thalamus. J Neurosci 14:3511-3532.

Orban GA (1984) Neuronal operations in the visual cortex. In: Studies of brain function XI (Braitenberg V, ed). New York: Springer.

Pouget A, Zhang K, Latham PE (1998) Statistically efficient estimation using population coding. Neural Comput 10:373-401.

Ruiz S, Crespo P, Romo R (1995) Representation of moving tactile stimuli in the somatic sensory cortex of awake monkeys. J Neurophysiol 38:1403-1420.

Salinas E, Abbott LF (1994) Vector reconstruction from firing rates. J Comp Neurosci 1:89-107.

Schöner G, Kopecz K, Erlhagen W (1997) The dynamic neural field theory of motor programming: arm and eye movements. In: Selforganization, computational maps and motor control (Morasso P, Sanguineti V, eds), pp 271-310. Amsterdam: Elsevier Science.

Seung HS, Sompolinsky H (1993) Simple models for reading neuronal population codes. Proc Natl Acad Sci USA 90:10749-10753.

Sillito AM, Grieve KL, Jones HE, Cudeiro J, Davis J (1995) Visual cortical mechanisms detecting focal orientation discontinuities. Nature 378:492-496.

Steinmetz MA, Motter BC, Duffy CJ, Mountcastle VB (1987) Functional properties of parietal visual neurons: radial organization of directionalities within the visual field. J Neurosci 7:177-191.

Sugihara T, Edelman S, Tanaka K (1998) Representation of objective similarity among three-dimensional shapes in the monkey. Biol Cybern $78: 1-7$.

Szulborski RG, Palmer LA (1990) The two-dimensional spatial structure of nonlinear subunits in the RFs of complex cells. Vision Res 30:249-254.

Tolhurst DJ, Heeger DJ (1997) Comparison of contrast-normalization and threshold models of the responses of simple cells in cat striate cortex. Vis Neurosci 14:293-309.

Vogels R (1990) Population coding of stimulus orientation by striate cortical cells. Biol Cybern 64:24-31.

Westheimer G (1990) Simultaneous orientation contrast for lines in the human fovea. Vision Res 30:1913-1921.

Wilson HR, Cowan JD (1973) A mathematical theory of the functional dynamics of cortical and thalamic nervous tissue. Kybernetik 13:55-80.

Wilson MA, McNaughton B (1993) Dynamics of the hippocampal ensemble code for space. Science 256:1055-1058.

Young MP, Yamane S (1992) Sparse population coding of faces in the infero-temporal cortex. Science 256:1327-1331.

Zemel RS, Dayan P, Pouget A (1998) Probabilistic interpretation of population codes. Neural Comput 10:403-430.

Zhang K (1996) Representation of spatial orientation by the intrinsic dynamics of head direction cell ensemble: a theory. J Neurosci 16:2112-2126.

Zhang K, Ginzburg I, McNaughton BL, Sejnowski TJ (1998) Interpreting neuronal population activity by reconstruction: unified framework with application to hippocampal place cells. J Neurophysiol 79:1017-1044.

Zohary E (1992) Population coding of visual stimuli by cortical neurons tuned to more than one dimension. Biol Cybern 66:265-272. 